\title{
NOTES ON FOUR SURGICAL CASES
}

\author{
By McNEILL LOVE, M.S., F.R.C.S. \\ (Surgeon, Royal Northern Hospital, etc.)
}

CASE I

History.-Male 64, occupation gardener, attended Hospital with a swelling on the back of the right wrist, which he had noticed for the past two years. Beyond slight occasional aching, which he volunteered was worse in damp weather, he presented no other symptom.

Examination.-Following the usual routine, which should be adopted for the examination of any swelling, the physical signs were as follows:-

Site-over the posterior aspect of the wrist joint, slightly to the radial side (Fig. I). Size-a pigeon's egg.

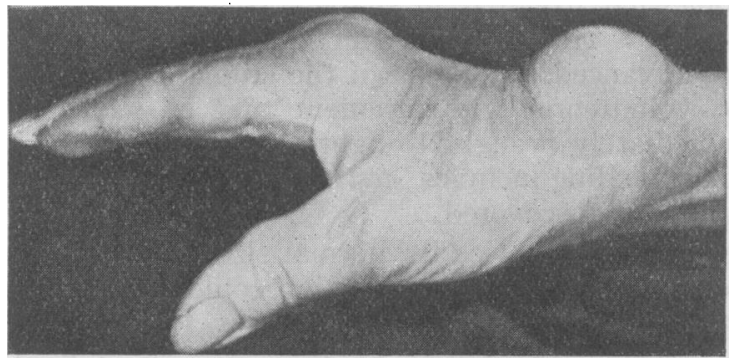

Fro. I.-Baker's cyst, following osteo-arthritis of the wrist joint.

[Reproduced from Bailey and Love's Short Practice of Surgery, by permission of $H$. $K$. Lewis \& Co.]

Shape-ovoid.

Attachments-not attached to the skin or any tendon, but deep aspect fixed to the capsule of the wrist joint.

Consistency - fluctuation obvious, and the swelling became more tense when the wrist joint was flexed.

Sensitivity-painless.

Passive movements of the wrist joint disclosed well-marked grating, suggesting an underlying condition of osteo-arthritis. This was supported by his work, as a lifelong occupation involving much digging could justifiably be considered as imposing "unfair wear and tear" on his right wrist. A radiograph confirmed the diagnosis of osteo-arthritis.

\section{Differential Diagnosis.}

Charcot's joint-which was suggested by the painlessness of the condition and obvious crepitus. However, advanced cases of osteo-arthritis are sometimes surprisingly painless, and examination of the central nervous system showed no evidence of syringomyelia (such as wasting of the muscles of both hands, scoliosis, unequal pupils or increased knee jerks). Nevertheless, this diagnosis was suggested by several candidates at a recent examination.

Simple gangloan.- The swelling was much larger than a typical simple ganglion, and showed no attachment to any tendon. Moreover, there would be no connection between a simple ganglion and the underlying osteo-arthritis.

Treatment.-The condition caused no disability, so a leather support was provided for the wrist, in order to retard further degeneration of the joint.

Note.-Morrant Baker's cysts occur most commonly in connection with the knee joint. They are associated with intermittent effusions, such as occur with osteo-arthritis and Charcot's disease. They are due to herniation of the synovial membrane through the capsule of the joint, and, as in the case of diverticulosis of the colon, this protrusion is liable to occur along the blood vessels. 
A Baker's cyst of the knee joint usually appears in the popliteal fossa, and it may be difficult to distinguish one from a semimembranosus bursa. Occasionally they track under muscles and pop out through an intermuscular septum some inches from the joint, and unless the clinician is aware of this migration he may be puzzled regarding the diagnosis. Morrant Baker emphasised this feature in his original paper.

\section{CASE II}

History.-Male aged I9 presented himself with symptoms of epigastric discomfort and increasing lassitude of about three months' duration, some nausea but no vomiting, except on one occasion when he took a double gin to relieve his symptoms. (N.B.- there is an increasing amount of ersatz gin on the market!) He stated that his parents had told him that he was looking unwell, and they insisted that he should obtain medical advice.

Examination.-General appearance somewhat pale and languid. Abdominal examination revealed a mass in the epigastrium nearly the size of a fist. The swelling was more in evidence on the left side, and somewhat irregular in shape. It was fixed to the posterior abdominal wall, and firm in consistency. He complained of slight tenderness on palpation. In spite of his age the possibility of advanced carcinoma of the stomach immediately came to mind, but at this juncture he had volunteered the statement that he had "something wrong below." Further examination immediately brought to light a swelling of the right testicle. A routine examination of a testicular swelling includes "testis, tunica, cord and patient," and adopting this routine the following signs were discovered.

Testes-the testicle was about twice its normal size, and it was, irregular in shape, with areas of altered consistency. No testicular sensation could be obtained, but the swelling was tender. Epididymis not palpable.

Tunica vaginalis - contained about two or three ounces of fluid, which was not easily transilluminable. This feature suggested a haematocele rather than a hydrocele.

Spermatic cord-obvious regular thickening of the right cord, due to increased vascularity and hypertrophy of the cremaster muscle.

Patient-as mentioned above, a fixed mass was present in epigastrium. Supraclavicular glands were not enlarged, and there was no clinical evidence of any other metastases.

It was obvious that the case was one of neoplasm of the testicle, and that there was no alternative diagnosis worthy of consideration. The age of the patient was younger than is usual for this condition, but it must be remembered that carcinoma of the testicle (and rectum) not infrequently arises comparatively early in life.

Treatment.-Owing to the fixity of the lumbar glands, radical removal of the testicle, cord, lymphatics and glands was out of the question. Orchidectomy was performed, with removal of the cord as high as the external ring, in order to prevent further dissemination and to forestall fungation. Macroscopically the testes was replaced by a soft, haemorrhagic growth, with areas of degeneration. The growth had sprouted through the tunica albuginea, and oozed into the tunica vaginalis, hence the haematocele. A radiograph of the chest was normal. Microscopical

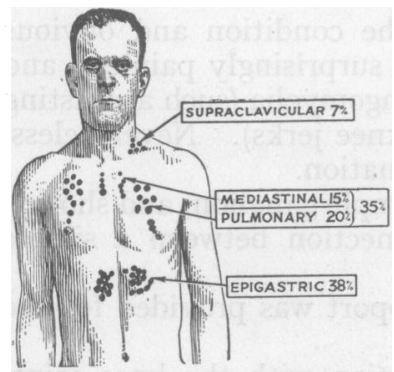

FIG. 2.-Distribution of secondary deposits following teratomata of the testicle.

[Reproduced from Bailey and Love's Short Practice of Surgery, by permission of $H K$ Lowis \& Co.] section proved the tumour to be a seminoma. Deep X-ray therapy is being applied to the glands, which responded almost immediately.

Note.-Malignant neoplasms of the testicle are uncommon, but a fixed epigastric mass in the male should always lead to descent of the trousers. Some patients, as in this case, are unduly reluctant to own to any abnormal condition of their genital organs.

Seminoma $(50 \%)$ is the commonest form of malignant disease of the testicle, although teratoma $(49 \%)$ is not far behind. The odd I \% includes sarcoma and chorio-epethelioma. Seminoma presents a better prognosis than teratoma, in that the secondaries are radio-sensitive, and they are limited for some time to the lumbar glands. In addition, teromata are radio-resistant and disseminate more widely, secondaries in the lung being common (Fig. 2). 


\section{CASE III}

A medical friend of mine telephoned me to say that he had under his care a woman, aged 37, whose abdomen was becoming greatly distended and whose general health was deteriorating. There were no acute symptoms of any kind, but would I take her into hospital under my care for investigation? I replied that I would be pleased to arrange for a bed in a few days' time. Three days later came another message to say that the patient was becoming drowsy, and her condition was giving rise to anxiety, so would we expedite admission? She was admitted the following morning.

History.-The patient was well nourished and sufficiently alert to give a brief history. Her chief complaint was that for about three months her abdomen had steadily increased in size, but she had felt well until about ten days before admission. During this period she had vomited occasionally, became short of breath, and a sensation of epigastric discomfort had developed. She had become increasingly drowsy and had taken to her bed.

Examination.- The abdomen was grossly distended. Palpation and percussion indicated that this was due to enormous enlargement of the liver, which was somewhat irregular and appeared to fill the whole abdomen. The spleen was not clinically enlarged, no obvious glands were present and no ascites could be detected. Examination by a physician revealed no abnormality in the heart or lungs. A catheter specimen withdrew some dark-coloured urine. The colour was thought to be due to blood, but ordinary tests failed to confirm this, so it was sent to the laboratory for examination.

A tentative diagnosis was made of secondary carcinoma of the liver, and the gall bladder or stomach were considered as possible candidates for the primary latent growth.

Twenty-four hours after admission the patient suddenly died. Post-mortem examination revealed a liver which weighed over $200 \mathrm{oz}$. (the normal weight being about $45 \mathrm{oz}$. for a female). The liver was almost entirely replaced by melanotic deposits, which characteristically varied in colour, some deposits being almost coal-black, and others nearly snow-white. The excessive destruction of the liver thus explained the cholaemic symptoms. Beyond a few ounces of free fluid there was no other abnormality in the peritoneal cavity.

Two small melanomatous deposits were present in the heart muscle, and a routine examination of the remainder of the body was negative. The question then arose as to the situation of the primary growth. Secondary deposits almost exclusively in the liver immediately suggested the uveal tract, but the absence of visual disturbance rendered this suggestion an unlikely one.

Examination of the nail beds, anal margin and nipples was negative, and no mole could be seen on the body. As a last resort a meticulous examination was made of the scalp, and in the right parietal area a small mole was discovered, smaller than a silver threepenny piece. The surface showed evidence of ulceration, and subsequent microscopic examination proved its malignancy. The pathologist's report on the urine was "melanuria."

Note-Melanomata malignum are amongst the most malignant of all tumours. This case well illustrates the fact that the primary tumour may be exceedingly small, whereas secondary deposits are enormous. Moreover, the variation in the colour of the secondary deposits is frequently noted. Ulceration and consequent bleeding is usually the first evidence that a mole has become malignant, and these symptoms are only too often a virtual death-warrant for the patient. Melanomata are radio-resistant, and, if anatomical conditions permit, the

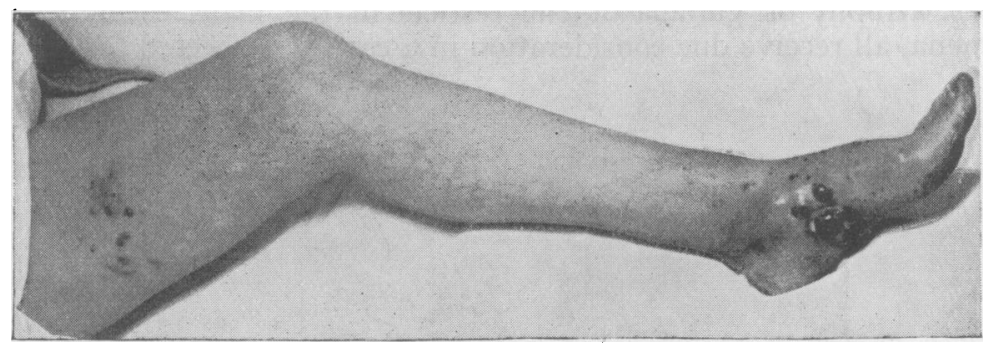

Fig. 3.-Melanoma of the foot with intermediate deposits in the lymphatics of the thigh. [Reproduced from Bailey and Love's Short Practice of Surgery, by permission of H. K. Lewis \& Co.] 
only treatment which holds out any hope is that advocated by Howarth Pringle of Newcastle. This consists in excision of the primary growth, a strip of deep fascia so as to include the intermediate lymphatic vessels, and the regional lymphatic glands all in one piece. Unless intermediate lymphatics are excised secondary deposits occur in the vessels and appear as pigmented, subcutaneous nodules (Fig. 3). This extensive operation is usually only applicable to a melanoma affecting a limb, and even then dissemination has probably spread beyond the operation field.

\section{CASE IV}

A lady, aged 62, was recently sent to me on account of a swelling in the lower part of her neck on the left side. It was thought to be either an enlarged lymphatic gland, or possibly a swelling in connection with the left lobe of the thyroid gland.

History.-The patient stated that she first noticed the swelling about three months previously, and that it had painlessly but steadily increased in size. She was a prominent Church worker, and her main complaint was that her voice had recently altered, so that when she sang she could not control her voice, much to her own embarrassment, and also to the discomfort of those within earshot.

Examination.-An obvious swelling was present, about the size of a walnut, in the substance of the left sterno-mastoid muscle. The situation was confirmed by the fact that active contraction of the muscle fixed the lump. The swelling was painless, and slightly adherent to the skin. These physical signs suggested a gumma of the sterno-mastoid muscle, which is a classical site for such a condition. The commonest site for clinical evidence of tertiary syphilis is the mouth, and the first four degrees of chronic superficial glossitis were clearly evident. They are-enlargement of papillae, leucoplakia, red glazed tongue due to desquamation, and scarring owing to submucous fibrosis. As yet, carcinoma had not developed. Laryngoscopic examination revealed some infiltration of the vocal cords, which explained the vocal disability.

Examination of the blood revealed negative a W.R., but the clinical evidence was so suggestive that a diagnosis of tertiary syphilis was made with confidence. It should be remembered that the W.R. is only positive in about $85 \%$ of cases of tertiary syphilis, so a negative result does not necessarily rule out the possibility that a doubtful swelling may be a gumma.

Antisyphilitic treatment has been instituted, and a good prognosis is to be expected. As there is no destruction of the vocal cords it is hoped that she will be readmitted to the choir when potassium iodide has exerted its usual beneficial effects.

Note.-Gummata are becoming increasingly rare, and for this reason they are apt to be overlooked, or misdiagnosed. If tertiary syphilis is suspected, confirmatory evidence is often found in the buccal cavity, where any of the following conditions may be found.

(i) Any stage of chronic superficial glossitis.

(ii) Chronic interstitial (parenchymatous) glossitis.

(iii) Scarring of the soft palate.

(iv) Perforation of the hard palate.

(v) Gumma or gummatous ulcer of the tongue, tonsil, palate or pharynx.

(vi) Hemiatrophy, due to involvement of the hypoglossal nerve by basal meningitis.

In addition, tissue paper, scars (silvery, supple and serpiginous), especially around and below the knees, atrophy or gumma of the testicle in the male, cardio-vascular signs and nervous phenomena, all receive due consideration in a case of suspected tertiary syphilis. 